\title{
PERFORMANCE ANALYSIS OF AL-FEC RAPTOR CODE OVER 3GPP EMBMS NETWORK
}

\author{
Avani U Pandya ${ }^{1}$, Sameer D Trapasiya ${ }^{2}$, Santhi S Chinnam ${ }^{3}$ \\ ${ }^{1}$ M.E. Research Scholar, ${ }^{2}$ Assistant Professor, Department of Electronics \& Communication Engineering, \\ G.H. Patel College of Engineering \& Technology.,Gujarat,India, \\ ${ }^{3}$ Telecom Engineer, $4 G$ RAN \& Device Validation, Rancore Technologies Pvt Ltd, Mumbai, India, \\ avani.pandya03@gmail.com, sameertrapasiya@gmail.com,swaroop419@gmail.com
}

\begin{abstract}
Long Term Evolution (LTE) is the current standard for mobile networks based on Third Generation Partnership Project. LTE includes enhanced multimedia broadcast and multicast services (MBMS), also called as Evolved multimedia broadcast and multicast services (eMBMS) where the same content is transmitted to multiple users in one specific area. eMBMS is a new function defined in $3 G P P$ Release 8 specification that supports the delivery of content and streaming to group users into LTE mobile networks. In LTE an important point of demanding multimedia services is to improve the robustness against packet losses. In this direction, in order to support effective point-to-multipoint download and streaming delivery, 3GPP has included an Application Layer Forward Error Correction (AL-FEC) scheme in the standard eMBMS. The standard AL-FEC system is based on systematic, fountain Raptor codes. Raptor coding is very useful in case of packet loss during transmission as it recover all data back from insufficient data at receiver terminal .In our work, in response to the emergence of an enhanced AL-FEC scheme, a raptor code has been implemented and performance is evaluated and the simulation results are obtained.
\end{abstract}

Index Terms: long term evolution; multimedia broadcast multicast services; forward error correction; raptor codes

\section{INTRODUCTION}

Nowadays there is a significant demand for multimedia services over wireless networks due to the explosive growth of the multimedia internet applications and dramatic increase in mobile wireless access. It is, therefore, foreseen that the wireless systems will have to support applications with increased complexity and tighter performance requirements, such as real-time video streaming. Furthermore, it is expected that popular content is streamed not just to a single user, but to multiple users attempting to access the same content at the same time. This is addressed by standardization bodies through introduction of a point-to-multipoint service enhanced Multimedia Broadcast Multicast Service (eMBMS), a resource-efficient transmission scheme targeting simultaneous distribution of multimedia content to many user devices within a serving area, over a single set of Core Network and Radio resources. So, Multimedia Broadcast and Multicast Service (MBMS) has been standardized as a key feature in Third Generation Partnership Project(3GPP) systems to broadcast and multicast multimedia content to multiple mobile subscribers via MBMS radio bearer service. MBMS is a point-to-multipoint (PTM) Standard, whose further evolvement and enrichment attracts nowadays widespread interest.

Long Term Evolution (LTE) has been designed to support only packet-switched services. Long Term Evolution (LTE) provides both the transmission mode single-cell MBMS, MBMS services which are transmitted in a single cell and multi-cellular evolved MBMS transmission mode, providing synchronous MBMS transmission from multiple cells, also known as multicast / broadcast single frequency network mode of transmission. To transmit the same data to multiple recipients allows network resources to be shared. MBMS extends the existing architecture with the introduction of 3GPP MBMS bearer service and MBMS user services. MBMS user services are constructed above the MBMS bearer service. For the delivery of MBMS-based services, 3GPP defines three functional layers. The first layer, called Bearers, provides a mechanism to data transmission over IP. Bearers based on point-to-multipoint data transmission (MBMS bearers), which can be used in conjunction with point-to-point transmission. The second layer is called delivery method which offers two modes of content delivery: download method of discrete objects and the streaming method providing continuous media. Delivery also provides reliability with FEC. The third layer (User Service/Application) enables applications to the end-user and allows him to activate or deactivate the service.

Generally an MBMS session includes the following three phases.

1. User Service Discovery phase: MBMS services are advertised to the end user using 2-way point-to-point TCP-IP- 
based communication or 1-way point-to-multipoint UDP-IPbased transmission.

2. Delivery phase: Multimedia content is delivered (either streaming or download mode) using 1-way point-tomultipoint UDP-IP transmission.

3. Phase after delivery: A user can report on the quality of the content received or request a repair service files (if the download delivery method) using 2-way point-to-point TCPIP communication.

During the delivery, a UDP packet can be eliminated by the physical layer, if bit errors cannot be corrected, and can be lost due to, for example, network congestion or hardware failure. As there is no feedback channel in the delivery phase of eMBMS, ARQ-based protocols cannot be used as those ARQ receivers use a feedback channel to the sender for requesting a retransmission of lost packets.

Another approach is to use Forward Error Correction (FEC) Codes. In order to analyze the performance of Forward Error Correcting (FEC) algorithms, we need to define a channel model. Binary Erasure Channel (BEC) was first introduced by Peter Elias of MIT in 1955[2]. It has been considered as too theoretic to draw attention, until the last few years when Internet became popular. Binary Erasure channel is defined as "a symbol either arrives to the destination, without any error or is erased and never received". Therefore, the receiver has no idea about the bit transmitted with a certain probability $\mathrm{p}$, and this is exactly sure of the bit transmitted with a probability 1-p. According to Shannon, BEC capacity is 1-p, which means that the size of the alphabet of $2 \mathrm{k}$, where $\mathrm{k}$ is the number of bits in the alphabet, no more than $(1-\mathrm{p}) \mathrm{k}$ bits/ symbols can be communicated reliably to the binary erasure channel. Automatic Repeat Request (ARQ) schemes have so long been used as a classical approach to solve the reliable communication problem [3]. In addition, any feedback from the receiver to the sender will not increase the channel capacity and reliable communication must be possible at this rate. ARQ works well for point-to-point transmission and has also been an effective tool for reliable point-to-multipoint transmission. However, when the number of receivers increases, ARQ reveals its limits. A major limitation is the problem of feedback implosion that occurs when too many receivers are transmitting to the sender. Another problem is that, for a loss rate of data packets, and a set of receivers experiencing losses, the probability that each data packet must be retransmitted rapidly approaching unity as the number of receivers becomes large. In other words, a high average number of transmissions required per packet. However, an excessive number of feedbacks are used in the case of erasures the wasting of bandwidth, network overload and intolerable delays occur.

Forward Error Correction (FEC) codes exist as Reed-Solomon codes, which can recover the $\mathrm{K}$ source symbols from the coded symbols of all $\mathrm{K}$ and $\mathrm{N}$ the total number of symbols transmitted. However, the rate $\mathrm{R}=\mathrm{K} / \mathrm{N}$ should be determined in accordance with the erasure probability $\mathrm{p}$, before transmission. If $\mathrm{p}$ changes or $\mathrm{p}$ is less or greater than expected, it will cause problems at the decoder side or will result in a lower transmission rate achievable. Another disadvantage of fixed powerful FEC codes is the high cost of decoding and encoding, for example, the Reed-Solomon codes have an encoding cost of $\mathrm{K}(\mathrm{N}-\mathrm{K}) \log 2 \mathrm{~N}$ [4].

Next is Low density parity check codes which were invented by Gallager in the 1960s. For each destination node, the sum of the values of its neighboring variable nodes must be zero. They consist of sparse generator matrices with reduced costs decoding when accompanied by Belief Propagation (BP). Some of these codes are very close to Shannon limit. However, they do not have a fast encoding algorithm, even if some approaches have been proposed, often running in linear time. The use of a code block, it is necessary to estimate the probability $\mathrm{p}$ of the BEC and adjust the encoder with this amount of redundancy.

Tornado codes have been developed for this purpose as an extension of LDPC codes with reduced complexity of encoding [5]. They can manage the rate adjustment, but not yet effective especially when the channel is subject to frequent changes.

Fountain Codes are a new class of codes designed and ideally suited for reliable transmission of data over an erasure channel with unknown erasure probability [6]. A fountain code has properties similar to a water Fountain which can be thought as an infinite supply of water drops. Anyone who wants to collect the water drops holds a bucket under the fountain. When enough water is collected, the bucket is removed. Similarly with a digital source, a client gets encoded packets from one or more servers and packages once enough are obtained, the client can reconstruct the original file, which packets are obtained should not matter. They are rate-less in the sense that for a given message, the encoder can produce potentially infinite number of output symbols. Output symbols can be bits or more general bit sequences. However, random linear Fountain Codes have encoding complexity of $\mathrm{O}$ (N2) and decoding complexity of $\mathrm{O}(\mathrm{N} 3)$ which makes them impractical for nowadays applications.

Luby Transform (LT) codes have been proposed by Michael Luby [7] to reduce the encoding and decoding complexity of random linear Fountain Codes while maintaining the small overhead. With a good choice of degree distribution, i.e. the distributions of the edges in the Tanner graph, LT codes can come arbitrarily close to channel capacity with certain decoder reliability and logarithmically increasing encoding and decoding costs. In order to reduce the complexity even more, we can decrease the reliability of the decoder. Thus, we would have a reduced degree distribution resulting linear time encoding and decoding complexity. However, the decoder 
cannot decode all the input symbols with the lower degree distribution for the same overhead constraint. Therefore, utilizing an erasure correcting pre-code would then correct the erasures arising from the weakened decoder. If the pre-code is a linear time block code, like an LDPC code, Raptor Codes provide marvelous encoding and decoding speeds while providing near optimal performance for the BEC [8].

Raptor codes are an extension of the other part of LT codes combined with a system of pre-coding. The design and degree distribution pre-coding is the heart of Raptor codes. Instead, the media data is protected using the application layer FEC with Raptor codes. To systematically increase the reliability of the transmission, an application layer FEC code can be used.

The rest of this paper is organized as follows: in Section II we provide an overview of the 3GPP AL-FEC eMBMS delivery framework and Section III presents a detailed description of the examined AL-FEC scheme. In Section IV we present the simulation environment and the conducted experimental results. Finally, in Section V we draw our conclusions and we describe some possible future steps.

\section{EMBMS PROTOCOL STACK}

\subsection{GPP AL-FEC eMBMS DELIVERY}

The 3GPP standard multicast services, MBMS mentioned [1], is a service of point-to-multipoint way in which data is transmitted from a single source to a group of several mobile terminals in an area of specific service. 3GPP defines two delivery methods namely, downloading and streaming. eMBMS user plane stack of these delivery methods is illustrated in Fig. 1.

1) MBMS Streaming Delivery Protocols Stack: The purpose of the MBMS streaming delivery method is to deliver continuous multimedia data (i.e. speech, audio, video) over an MBMS bearer. MBMS makes use of the most advanced multimedia codecs such as H.264 for video applications and enhanced Advanced Audio Coding (AAC) for audio applications. Realtime transport protocol (RTP) is the transport protocol for MBMS streaming delivery. RTP provides means for sending real-time or streaming data over user datagram protocol (UDP), the resulting UDP flows are mapped to MBMS IP multicast bearers. Then IP packets are processed in the Packet Data Convergence Protocol (PDCP) layer where for example header compression might be applied. In the Radio Link Control (RLC) the resulting PDCP- Protocol Data Units (PDUs), generally of arbitrary length, are mapped to fixed length RLC-PDUs. The RLC layer operates in unacknowledged mode as feedback links on the radio access network are not available for point-to-multipoint bearers. The RLC layer is responsible for mapping IP packets to RLC SDUs.Functions provided at the RLC layer are for example segmentation and reassembly, concatenation, padding, sequence numbering, reordering and out-of-sequence and duplication detection. The Medium Access Control (MAC) layer maps and multiplexes the RLC PDUs to the transport channel and selects the transport format depending on the instantaneous source rate. The MAC layer and physical layer appropriately adapt the RLC-PDU to the expected transmission conditions by applying, among others; channel coding, power and resource assignment, and modulation. [1]

\section{2).MBMS Download (File) Delivery Protocols Stack: MBMS} download delivery method aims to distribute discrete objects (e.g. files) by means of a MBMS download session. Download method uses the File deLivery over Unidirectional Transport (FLUTE) protocol when delivering content over MBMS bearers. FLUTE is built on top of the Asynchronous Layered Coding (ALC) protocol instantiation. ALC combines the Layered Coding Transport (LCT) building block and the FEC building block to provide reliable asynchronous delivery of content to an unlimited number of concurrent receivers from a single sender. A detailed description of the FLUTE building block structure can be found in [1]. Thereafter, FLUTE is carried over UDP/IP, and is independent of the IP version and is forwarded to the Packet Data Convergence Protocol (PDCP) layer. The packets are then sent to the Radio Link Control (RLC) layer. The RLC Layer functions in unacknowledged mode. The RLC layer is responsible for mapping IP packets to RLC SDUs. The Media Access Control (MAC) Layer adds a 16 bit header to form a PDU, which is then sent in a transport block on the physical layer.

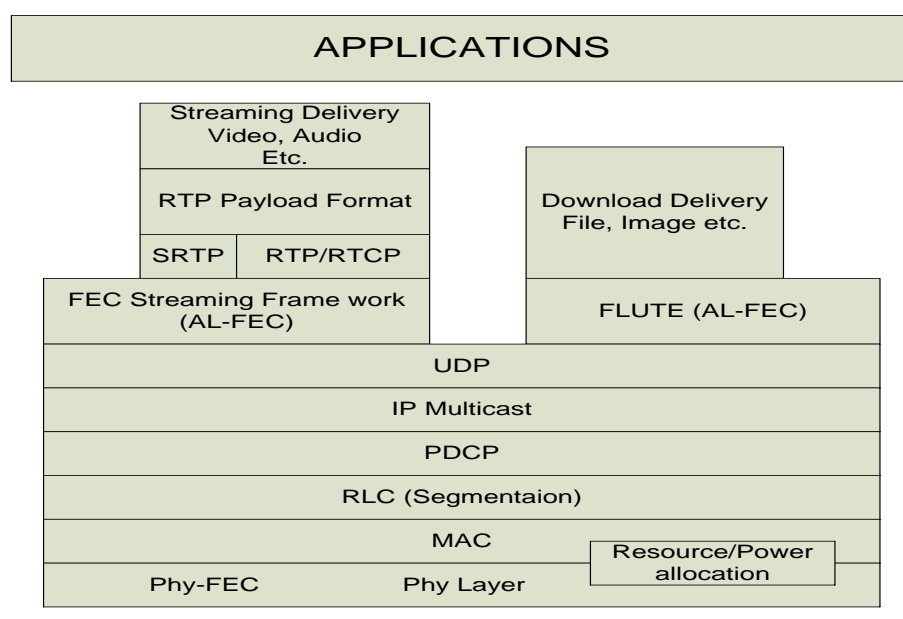

Figure 1: eMBMS protocol stack

\section{AL-FEC Scheme}

In this section we provide a detailed description of the 3GPP standardized Raptor code.

\subsection{RAPTOR CODES}

Raptor codes were introduced in [9]. The use of Raptor codes in the application layer of eMBMS was introduced to 3GPP 
aiming to improve service robustness against packet losses. Raptor codes are fountain codes, meaning that as many encoding symbols as desired can be generated by the encoder on-the-fly from the source symbols of a source block of data. Raptor codes are one of the first known classes of fountain codes with linear encoding and decoding time [9]. The systematic Raptor Encoder is used to generate repair symbols from a source block that consists of K source symbols [10].In preparation of the encoding; a certain amount of data is collected within a FEC source block. The data of a source block are further divided into $\mathrm{k}$ source symbols of a fixed symbol size. The decoder is able to recover the whole source block from any set of encoding symbols only slightly more in number than the source symbols. The Raptor code specified for MBMS is a systematic code producing $n$ encoding symbols $\mathrm{E}$ from $\mathrm{k}<\mathrm{n}$ source symbols $\mathrm{C}$, so as the original source symbols are within the stream of the transmitted symbols. This code can be viewed as the concatenation of several codes. The most inner code is a non-systematic Luby-Transform (LT) code [7] with 1 input symbols $F$, which provides the fountain property of the Raptor codes. This non-systematic Raptor code is not constructed by encoding the source symbols with the LT code, but by encoding the intermediate symbols generated by some outer high-rate block code. This means that the outer high rate block code generates the $\mathrm{F}$ intermediate symbols using $\mathrm{k}$ input symbols D. Finally, a systematic realization of the code is obtained by applying some pre-processing to the $\mathrm{k}$ source symbols $\mathrm{C}$ such that the input symbols $\mathrm{D}$ to the nonsystematic Raptor code are obtained [11]. Considering the performance of Raptor codes the most typical comparison is that to an ideal fountain code. An ideal fountain code can produce from any number $\mathrm{k}$ of source symbols any number $\mathrm{m}$ of repair symbols with the property that any combination of $\mathrm{k}$ of the $\mathrm{k}+\mathrm{m}$ encoding symbols is sufficient for the recovery of the $\mathrm{k}$ source symbols. That is the point of the most important differentiation between an ideal fountain code and the standardized Raptor code. While an ideal code has zero reception overhead i.e., the number of received symbols needed to decode the source symbols is exactly the number of source symbols, the Raptor code has a performance close to that property. The performance of an AL-FEC code can be described by the decoding failure probability of the code. The study presented in [12] describes the decoding failure probability of Raptor code as a function of the source block size and the received symbols. In fact, the inefficiency of the Raptor code can accurately be modeled by (1) [12]

$$
p_{f_{R}}(n, k)=\left\{\begin{array}{cc}
1 & \text { if } n<k \\
0.85 \times 0.567^{n-k} & \text { if } n \geq k
\end{array}\right.
$$

In (1), pfR(n,k) denotes the decoding failure probability of the Raptor code if the source block size is $k$ symbols and $n$ encoding symbols have been received. Failure probability decreases exponentially when the number of received encoding symbols increases. Moreover, a crucial point for the robustness of an AL-FEC protected delivery session is the transmission overhead. The transmission overhead is defined as the amount of redundant information divided by the amount of source data and is equal to the fraction $(\mathrm{N}-\mathrm{K}) / \mathrm{K}$ in terms of percentage. In this fraction, $\mathrm{N}$ denotes the number of transmitted packets and $\mathrm{K}$ denotes the number of the source packets.

1) 3GPP Raptor Encoding Process: Raptor codes [10] are serially concatenated codes with a pre-code as the outer code and the LT code [1] as the inner code. Pre-code itself is also a serially concatenated code, which uses an LDPC code and a code with dense parity (Gray code) check matrix as the outer code and the inner code, respectively. For Raptor Encoder source object is divided into $\mathrm{Z}>=1$ number of source blocks. Each source block has K source symbols of size T bytes each. Each source block is encoded independently from the next Block. The source block construction is specified in 3GPP 26.346 [1]. The systematic Raptor codes generate encoding symbols which contain $\mathrm{K}$ source symbols plus repair symbols. In this systematic Raptor code, $\mathrm{K}$ original input symbols are first encoded to $\mathrm{L}=\mathrm{K}+\mathrm{S}+\mathrm{H}$ intermediate symbols by precode. Here, $\mathrm{S}$ and $\mathrm{H}$ are the amount of redundancy added by the outer and the inner codes of the pre-code, respectively. And then, we can generate any number of encoding symbols as needed with those intermediate symbols. The intermediate symbols are related to the source symbols by a set of source symbol triples. The encoding process is to produce repair symbols by bit-wise XORing specific source symbols. The whole process is divided in following steps.

Step 1:- The first step in encoding can be performed by generating an $\mathrm{LxL}$ encoding matrix, $\mathrm{A}$ to calculate $\mathrm{L}$ intermediate symbols as [10].

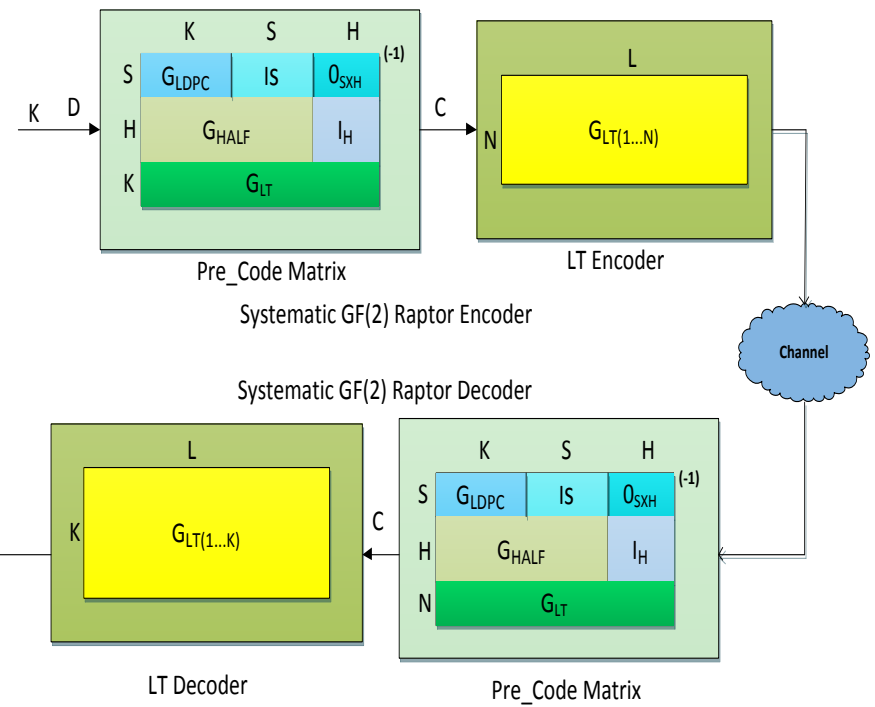

Figure 2: Block diagram of Raptor encoder and decoder 


$$
C=A^{-1} D
$$

Where, C represents column vector of the $\mathrm{L}$ intermediate symbols, D represents column vector of $\mathrm{S}+\mathrm{H}$ zero symbols followed by the K source symbols.

The precode matrix A consists of several submatrices as shown in figure 2. In this matrix $\mathrm{A}$, the top $\mathrm{S}+\mathrm{H}$ rows represent the constraints of the pre-code on $\mathrm{C}$, and the bottom $\mathrm{K}$ rows, each corresponding to a source symbol of $\mathrm{D}$, represent the generator matrix of the LT code. (GLDPC) $\mathrm{S} \times \mathrm{K}$ and (HHa1f) $\mathrm{H} \times(\mathrm{S}+\mathrm{K})$ correspond to a LDPC check matrix and a high dense parity check matrix, respectively. I_S be the S x S identity matrix I_H be the H x H identity matrix 0_SxH be the $\mathrm{S} \times \mathrm{H}$ zero matrix and (GLT) $\mathrm{KxL}$ is a LT encoding matrix. (GLDPC)S X K and (HHa1f) H X (S+ K) are known on the both encoder and decoder side.

Step 2:-The second encoding step is to generate the repair symbols from $\mathrm{L}$ intermediate symbols using LT encoding process as LTEnc[K,C[0],C[1],..,C[L-1],(d,a,b)] [10] where $(d, a, b)$ represents triples for each symbol.

At the end of step 2 final transmitted streams are generated.

2) 3GPP Raptor Decoding Process: The N received symbols are input to the decoder where

$$
\mathrm{N}=\mathrm{K}+\mathrm{R}-\mathrm{Ls}
$$

Where $\mathrm{K}$ defines the no. of source symbols, $\mathrm{R}$ defines the repair symbols and Ls represent loss symbols.

Matrix A of (M x L) can be formed similar to the encoding process using received $\mathrm{N}$ symbols [10] where

$$
\mathrm{M}=\mathrm{S}+\mathrm{H}+\mathrm{N}
$$

Where $\mathrm{S}$ is LDPC symbols, $\mathrm{H}$ Half Symbols and $\mathrm{N}$ received symbols respectively. The matrix, $\mathrm{A}$ is a bit matrix that satisfies $\mathrm{A} \times \mathrm{C}=\mathrm{E}$ using matrix multiplication in $\mathrm{GF}$ (2). Intermediate symbols $\mathrm{C}$ can then be decoded if the bit matrix $\mathrm{A}$ is square $(\mathrm{L} \times \mathrm{L})$ and invertible. Since the number of received encoding symbols, $\mathrm{N}>\mathrm{K}$ in most cases, so following steps should be taken for decoding.

Step 1:- The first step in decoding is to convert ( $\mathrm{M} \times \mathrm{L}$ ) matrix $\mathrm{A}$ to an ( $\mathrm{L} \mathrm{x}$ L) matrix using Gaussian Elimination method [10].

Improved Gaussian elimination, consisting of row/column exchange and row Ex-OR, is used in the 3GPP Raptor decoding algorithm. In the decoding process, the original matrix A will be converted into an identity matrix. Besides, vector $\mathrm{C}$ and $\mathrm{D}$ change concurrently. Let $(\mathrm{N} \geq \mathrm{K})$ be the number of received encoding symbols and $\mathrm{M}=\mathrm{S}+\mathrm{H}+\mathrm{N}$. The vector $\mathrm{D}=(\mathrm{D}[0], \ldots, \mathrm{D}[\mathrm{M}-1])$ is the column vector of $\mathrm{M}$ symbols with values known to the receiver, where $\mathrm{D}[0], \ldots$ ,D $[\mathrm{S}+\mathrm{H}-1]$ are zero-valued symbols that correspond to LDPC and Half symbols, $\mathrm{D}[\mathrm{S}+\mathrm{H}], \ldots, \mathrm{D}[\mathrm{M}-1]$ are the received encoding symbols for the source symbols. When the original matrix A is converted into identity matrix successfully, we can get the intermediate symbols from D.

Before Gaussian elimination, we assume $\mathrm{C}[0]=0$, $\mathrm{C}[1]=1, \ldots \quad, \mathrm{C}[\mathrm{L}-1]=\mathrm{L}-1 \quad$ and $\mathrm{D}[0]=0, \mathrm{D}[1]=1, \ldots$ , $\mathrm{D}[\mathrm{M}-1]=\mathrm{M}-1$.initially. In the process of Gaussian elimination, the vectors $\mathrm{C}$ and $\mathrm{D}$ change concurrently with the changes of matrix A. The process abides by the rules as follows:

$>$ If the row $\mathrm{i}$ of $\mathbf{A}$ is exclusive-ORed into row $\mathrm{i}^{\prime}$, then symbol $\mathrm{D}[\mathrm{d}[\mathrm{i}]]$ is exclusive-ORed into symbols $\mathrm{D}\left[\mathrm{d}\left[\mathrm{i}^{\prime}\right]\right]$;

$>$ If the row $\mathrm{i}$ of $\mathbf{A}$ is exchanged with row $i^{\prime}$, then the value $d[i]$ is exchanged with the value $d[i$ '];

$>$ If the column $\mathbf{j}$ of $\mathbf{A}$ is exchanged with column $\mathrm{j}^{\prime}$, then the value $c[j]$ is exchanged with the value $c[j]$.

It is clear that $\mathrm{C}[\mathrm{c}[0]], \mathrm{C}[\mathrm{c}[1]], \ldots, \mathrm{C}[\mathrm{c}[\mathrm{L}-1]]=\mathrm{D}[\mathrm{d}[0]]$, $\mathrm{D}[\mathrm{d}[1]], \ldots, \mathrm{D}[\mathrm{d}[\mathrm{L}-1]]$ at the end of successful decoding. The process of converting A into identity matrix consists of four phases:

Phase I:-In the first phase of the Gaussian elimination, the matrix $\mathrm{A}$ is partitioned into submatrices, where I is an identity matrix $\mathrm{i} \times \mathrm{i}, \mathrm{O}$ is a matrix with all values zero, $\mathrm{U}$ is a matrix with u columns. An efficient algorithm, suggested in 3GPP MBMS standard, is helpful for choosing an appropriate row to do Gaussian elimination. The algorithm defines a graph structure of matrix V. Let the columns of $\mathrm{V}$ be the set of nodes and let the rows that have exactly two 1's in $\mathrm{V}$ be the set of edges that connect the associated nodes. We define component as follows: component in the graph is a set of nodes and edges such that there is a path between all pairs of nodes, the size of a component is the number of nodes in the component.

When $\mathrm{i}+\mathrm{u}=\mathrm{L}$, the first phase ends successfully and the matrix $\mathrm{V}$ disappears. If there are non-zero rows in $\mathrm{V}$ can be chosen in the first phase, we can use the efficient 3GPP standard as follows:

$>$ Let $r$ represents the minimum integer that at least one row has exactly $r$ 1's in $\boldsymbol{V}$. When $r \neq 2$, choose a row with exactly $r$ 1's in $\boldsymbol{V}$ with minimum original degree among all such rows;

$>$ When $r=2$, choose any row contained in the maximum size component [13] of the graph defined by $\boldsymbol{V}$.

Then the first row is exchanged with the chosen row of $\mathrm{V}$ and the arbitrary column, contained 1 in the chosen row, is exchanged with the first column. Besides, the columns contained remaining $(\mathrm{r}-1)$ 1's are exchanged with the last 
columns of V. After that, the chosen rows are exclusive-ORed into the rows of A below the chosen row that have a 1 in the first column of V. Accompanied by the process above, $\mathrm{i}$ is increased by 1 and $u$ is increased by (r-1). We carry out the first phase again until it ends successfully.

Phase II: - In the second phase, the submatrix U is partitioned into the first i rows, $\mathrm{U}_{-}$upper, and the remaining $(\mathrm{M}-\mathrm{i})$ rows, U_lower. If the rank of U_lower is u, Gaussian elimination is performed on it, and converts it into a matrix where the first $\mathrm{u}$ row is the identity matrix. Besides, the last (M-L) rows are discarded.

Phase III \& IV: - The task of the third and the fourth phase is to convert the U_upper into a matrix with all values zero.

Once the Gaussian Elimination is complete the bit matrix, A becomes $\{\mathrm{LxL}\}$ and invertible. The intermediate symbols $\mathrm{C}$ can then be obtained as

$$
\mathrm{C}=\mathrm{A}-1 \mathrm{D}
$$

Here D represents column vector of $\mathrm{S}+\mathrm{H}$ zero symbols followed by the $\mathrm{N}$ received symbols. The intermediate symbols, C is then passed to LT decoding to regenerate the $\mathrm{K}$ Source symbols. [10]

\section{PERFORMANCE EVALUATION OF RAPTOR}

\section{CODES:}

It is clear that the key points featuring the performance of an AL-FEC scheme are the decoding failure performance with respect to the number of additional symbols received and further, as a direct consequence of this aspect, the amount of the transmission redundancy required to confront different packet losses patterns.

To this direction, firstly we investigate the decoding performance compared to the reception overhead such a FEC code requires to successfully recover the protected data. Figure 3 presents the probability the FEC decoding process to fail in function to the number of additional symbols received, i.e., the reception overhead .For each reception overhead, the results are acquired from 10,000 experimental trials with symbols erased at random. Comparing the performance of the standardized Raptor FEC code for $\mathrm{K}=254$ each with case $\mathrm{N} 1=254(\mathrm{~K}), \mathrm{N} 2=127(\mathrm{~K} / 2), \mathrm{N} 3=93(\mathrm{~K} / 4)$.
Table -1: Comparison Table for RFC $5053 \mathrm{~K}=254$ for Latency 3

\begin{tabular}{|c|c|c|c|c|c|}
\hline $\begin{array}{c}\text { Sourc } \\
\text { e } \\
\text { Symb } \\
\text { ols } \\
(\mathbf{K})\end{array}$ & $\begin{array}{c}\text { Repair } \\
\text { Symbols } \\
(\mathbf{N})\end{array}$ & $\begin{array}{c}\text { Total } \\
\text { Symbols } \\
(\mathbf{K}+\mathbf{N})\end{array}$ & PbE & $\begin{array}{c}\text { Encod } \\
\text { ing }\end{array}$ & $\begin{array}{c}\text { Supp } \\
\text { ort } \\
\text { Cost } \\
\text { Pack } \\
\text { et }\end{array}$ \\
\hline 254 & $254(\mathrm{~K})$ & 508 & 0.163 & $50 \%$ & 49.4 \\
& & & 7 & & $\%$ \\
\hline 254 & $127(\mathrm{~K} / 2)$ & 381 & 0.142 & 33.33 & 32.54 \\
& & & 0 & $\%$ & $\%$ \\
\hline 254 & $64(\mathrm{~K} / 4)$ & 318 & 0.121 & 20.12 & 19.18 \\
& & & 3 & $\%$ & $\%$ \\
\hline
\end{tabular}

Here Encoding Cost= Repair Symbols $/$ Total Symbols. For Supporting Packet Loss we are noting all the values for Reception Overhead 3 (which resembles the latency). So we have to receive 3 more extra symbols which mean we have to receive $254+3=257$ symbols. or $\mathrm{N}=\mathrm{K}$ case, total 508 symbols we are transmitting. out of which we must receive 257 symbols, hence channel can drop at maximum (508-257 = 251 symbols).so allowable channel loss is Maximum dropped packets $/$ Total Transmitting symbols $=251 / 508=49.4 \%$ For $\mathrm{N}=\mathrm{K} / 4$ case, total 318 symbols we are transmitting. Out of which we must receive 257 symbols, hence channel can drop at maximum $(318-257=61$ symbols $)$ so allowable channel loss is Maximum dropped packets / Total Transmitting symbols $=61 / 318=19.18 \%$

For case N=K/4 64 Repair symbols were used which shows that decoding failure probability also decreases with less bandwidth utilization

From Table 1 it is clear that for worst channel condition $\mathrm{N}=\mathrm{K}$ case is useful as supporting Packet loss is $49.4 \%$ and for $\mathrm{N}=\mathrm{K} / 4$ case is useful for good channel condition as encoding cost and $\mathrm{PbE}$ are less compared to $\mathrm{N}=\mathrm{K}$. 


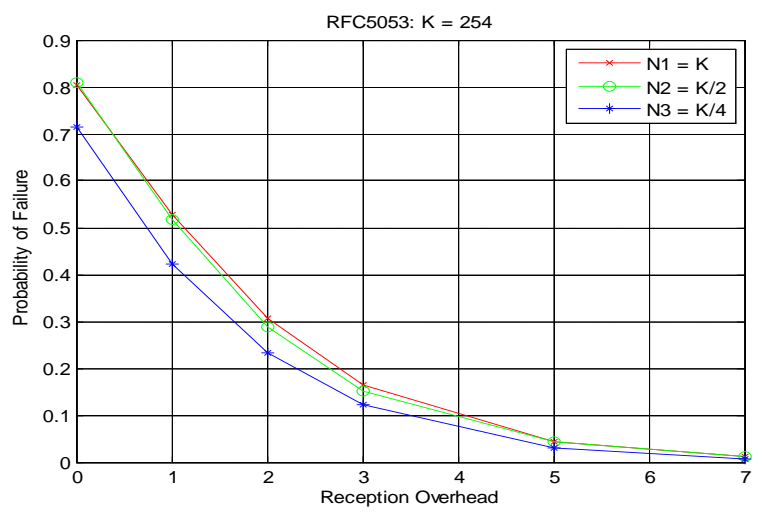

Figure 3: FEC decoding failure probability versus Reception overhead for $\mathrm{K}=254$

Comparing the three plotted curves behavior in fig 3 and fig 4 , we can immediately remark that a Raptor failure probability decreases exponentially with the growth of the number of additional FEC symbols.

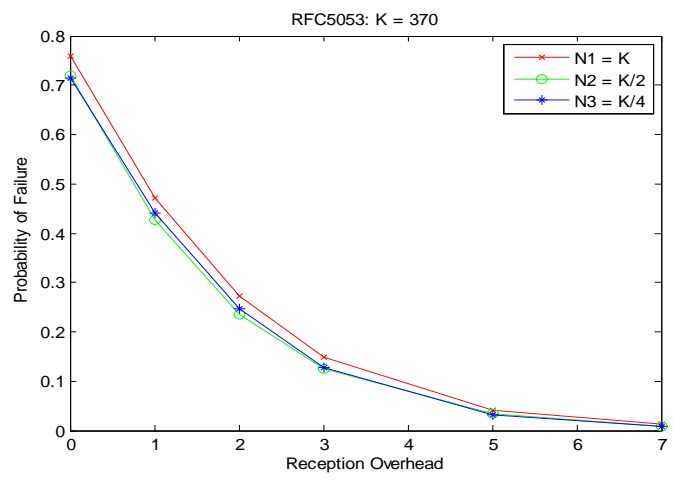

Figure 4: FEC decoding failure probability versus Reception overhead for $\mathrm{K}=370$

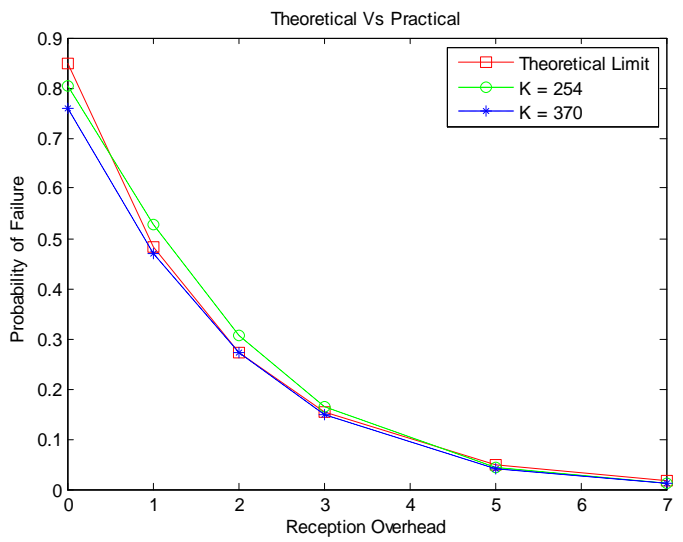

Figure 5: FEC decoding failure probability versus Reception overhead for Theoretical Vs Practical
Also as per equation (1) we have plotted curve as shown in fig 5 for theoretical Vs RFC 5053 (practical) which shows that we are approaching to the theoretical limit and even at some point we are getting better results than theoretical with lower decoding failure probability.

\section{CONCLUSION AND FUTURE WORK}

In this work we have implemented and then provided an extensive performance evaluation of AL-FEC based on the Raptor codes(IETF RFC 5053) provided by Digital Fountain to provide reliability against packet losses in 3GPP LTE eMBMS services. We have examined how the Raptor overhead varies during different network conditions and which is the optimal overhead that a multicast sender should introduce to the transmission to achieve successful delivery of the multimedia content in a PTM manner.

Some future actions that may result from this work may be possible; consideration is the design and evaluation of an adaptive algorithm which calculates the optimum FEC encoding process. This mechanism could be based on a system of feedback reports on network conditions, as proposed in the database and reports coding parameters. Finally, recently appeared AL-FEC RaptorQ scheme, could further enhance the research field of reliable multicast in mobile networks.

\section{ACKNOWLEDGEMENTS}

We would like to thank Yashesh Buch for providing such an interesting topic. We are also grateful to Harish Padi for valuable discussions and friendly support.

\section{REFERENCES:}

[1] 3GPP TS 26.346 V10.4.0.," Technical Specification Group Services and System Aspects: MBMS, Protocols and codecs", (Release 10), 2012.

[2] P. Elias. Coding for two noisy channels. in Proc. 3rd London Symposium on Information Theory, pages 61-76, 1955.

[3] Han Wang, "Hardware Design for LT Coding", $M S c$ Thesis,University of Delft, Holland, 2006.

[4] Berlekamp, E.R.: "Algebraic coding theory", McGrawHill, New York, 1968.

[5] M. Luby, M. Mitzenmacher, A. Shokrollahi, and D. Spielman, "Efficient erasure correcting codes", IEEE Trans. Inf. Theory, vol. 47, no. 2, pp. 569-584, Feb. 2001

[6] David J. C. MacKay, "Fountain Codes", Communications, IEEE proceedings vol. 152, no. 6, Dec. 2005, pp. $1062-1068$.

[7] M. Luby, "LT codes", Proc. 43rd Ann. IEEE Symp. On Foundations of Computer Science, Nov. 2002, pp. 271282.

[8] A. Shokrollahi, "Raptor Codes", in Proc. IEEE Int. Symp. Information Theory, Chicago, IL, Jun. /Jul. 2004, p. 36. 
[9] A. Shokrollahi, "Raptor codes," Information Theory, IEEE Transactions on, vol. 52, no. 6, pp. 2551-2567, June 2006.

[10] M. Luby, A. Shokrollahi, M. Watson, and T. Stockhammer, "Raptor forward error correction scheme for object delivery," September 2007, Internet Engineering Task Force, RFC 5053. Available at http://tools.ietf.org/html/rfc5053.

[11] Luby M, Watson M, Gasiba T, Stockhammer T, Xu W." Raptor codes for reliable download delivery in wireless broadcast systems." In Proc. of CCNC, 2006.

[12] T. Stockhammer, A. Shokrollahi, M. Watson, M. Luby, and T. Gasiba,"Application Layer Forward Error Correction for mobile multimedia broadcasting," Handbook of Mobile Broadcasting: DVB-H, DMB, ISDBT and Media Flo, CRC Press, pp. 239-280, 2008.

[13] S. Dasgupta, C. H. Papadimitriou, and U. V. Vazirani, “ Algorithms ," McGraw-Hill, July 18, 2006

\section{BIOGRAPHIES:}

Avani U Pandya has received the B.E (Electronics\& Communication Engineering) degree from North Gujarat University (Government Engineering College, Modasa) in 2007. She is currently a M.E (Communication) student (4th semester) in G .H. Patel College of Engineering \& Technology Gujarat from Gujarat Technological University, India. Her current research interests include 4G LTE eMBMS Network, Wireless communication

Prof Sameer D Trapasiya is Presently working in Electronics\& Communication Engineering Dept,G .H. Patel College of Engineering \& Technology., Gujarat.He has more than 6 years of experience in teaching. His current areas of interest are Cognitive radio, MIMO Communication., Cross layer optimization, Wireless Sensor Network.

Santhi S Chinnam is Presently Telecom Engineer in 4G RAN \& Device Validation at Rancore Technologies Pvt Ltd, Navi Mumbai. He has received his Mtech in Communications from IIT Roorkee 